\title{
CIENCIAMATRIA
}

Revista Interdisciplinaria de Humanidades, Educación, Ciencia y Tecnología

Año VIII. Vol. VIII. N¹4. Enero - Junio. 2022

Hecho el depósito de ley: pp201602FA4721

ISSN-L: 2542-3029; ISSN: 2610-802X

Universidad Nacional Experimental Francisco de Miranda (UNEFM). Santa Ana de Coro. Venezuela

Rously Eedyah Atencio-González; Elba Tatiana Peñaherrera-Martínez; Rudy Gabriela Yong-Zambrano Gabriel Alexander Lopez-Sanchez

DOI 10.35381/cm.v8i14.609

\section{La constitucionalidad de los derechos humanos en Ecuador}

The constitutionality of human rights in Ecuador

\author{
Rously Eedyah Atencio-González \\ uq.rouslyatencio@uniandes.edu.ec \\ Universidad Regional Autónoma de los Andes, Quevedo \\ Ecuador \\ https://orcid.org/0000-0001-6845-1631 \\ Elba Tatiana Peñaherrera-Martínez \\ dq.elbatpm37@uniandes.edu \\ Universidad Regional Autónoma de los Andes, Quevedo \\ Ecuador \\ https://orcid.org/0000-0002-2474-339X \\ Rudy Gabriela Yong-Zambrano \\ dq.rudygyz21@uniandes.edu.ec \\ Universidad Regional Autónoma de los Andes, Quevedo \\ Ecuador \\ https://orcid.org/0000-0002-7206-9379 \\ Gabriel Alexander Lopez-Sanchez \\ dq.gabrielals58@uniandes.edu.ec \\ Universidad Regional Autónoma de los Andes, Quevedo \\ Ecuador \\ https://orcid.org/0000-0003-3128-6948
}

Recibido: 15 de septiembre 2021

Revisado: 10 de noviembre 2021

Aprobado: 15 de diciembre 2021

Publicado: 01 de enero de 2022 


\author{
CIENCIAMATRIA \\ Revista Interdisciplinaria de Humanidades, Educación, Ciencia y Tecnología \\ Año VIII. Vol. VIII. №14. Enero - Junio. 2022 \\ Hecho el depósito de ley: pp201602FA4721 \\ ISSN-L: 2542-3029; ISSN: 2610-802X \\ Universidad Nacional Experimental Francisco de Miranda (UNEFM). Santa Ana de Coro. Venezuela
}

Rously Eedyah Atencio-González; Elba Tatiana Peñaherrera-Martínez; Rudy Gabriela Yong-Zambrano Gabriel Alexander Lopez-Sanchez

\title{
RESUMEN
}

El objetivo general de la presente investigación fue analizar la constitucionalidad de los derechos humanos en Ecuador. Desde el paradigma cuantitativo, se manejó para este tipo de estudio, documentos, textos legales, investigaciones científicas mediante la recolección, selección y análisis de los mismos. El diseño bibliográfico-documental se apoyó en los procesos lógicos y mentales. Se consideró el análisis de contenido de la información recopilada, lo que permitió la formulación de resultados. Además, se apoyó en el análisis, analítico-sintético, consistió en descomponer mentalmente el sistema estudiado en varios elementos para poder llegar a la obtención de nuevos conocimientos. Se concluye, sobre la importancia jurídica y social del derecho constitucional relacionado a la protección de los derechos humanos en el Ecuador y más allá de sus fronteras, gracias a los convenios firmados con los organismos internacionales, como garantista el Estado ha propiciado normas protectoras.

Descriptores: Derechos humanos; constitución; igualdad de oportunidades. (Tesauro UNESCO).

\begin{abstract}
The general objective of this research was to analyze the constitutionality of human rights in Ecuador. From the quantitative paradigm, it was managed for this type of study, documents, legal texts, scientific research through the collection, selection and analysis of them. The bibliographic-documentary design was based on logical and mental processes. The content analysis of the collected information was considered, which allowed the formulation of results. In addition, it was supported by the analytical-synthetic analysis, it consisted of mentally breaking down the studied system into several elements in order to obtain new knowledge. It is concluded on the legal and social importance of constitutional law related to the protection of human rights in Ecuador and beyond its borders, thanks to the agreements signed with international organizations, as a guarantor the State has promoted protective regulations.
\end{abstract}

Descriptors: Human rights; constitution; equal opportunities. (UNESCO Thesaurus) 
Rously Eedyah Atencio-González; Elba Tatiana Peñaherrera-Martínez; Rudy Gabriela Yong-Zambrano Gabriel Alexander Lopez-Sanchez

\section{INTRODUCCIÓN}

La constitucionalidad de los derechos humanos en Ecuador, constituyen las bases de protección de todos los ciudadanos que habitan en el País Andino, así como los que se encuentran fuera de la Nación. En tal sentido, Lema (2009, p. 13) indica, que los derechos humanos son:

Aquellos atributos, facultades o prerrogativas que tienen los seres humanos por el solo hecho de existir, han sido inspirados en los siguientes principios:

Dignidad: pensada como el valor que poseen todas las personas, cualquiera sea su origen social, cultural, económico, político o religioso.

Libertad: comprendida en el sentido de que el hombre es libre por naturaleza y que aquella libertad puede ser expresada en todos los aspectos de su vida. Igualdad: según la cual todos los seres humanos poseemos los mismos derechos independientemente de nuestras diferencias de origen.

Seguridad: bajo la reflexión de que todos nacimos libres y tenemos igualdad de derechos, por lo que quien niegue esos derechos universales, debe responder ante la justicia (la justicia social sólo se alcanza a través de la igualdad entre individuos).

En este sentido, la operatividad de los derechos humanos, se apoya en la Constitución y los órganos encargados de su fiel cumplimiento. Al respecto Sosa Salazar et al. (2019), manifiestan lo siguiente:

El estado de derechos, aquel donde la ley pasa a segundo plano y la constitución es realmente material y centro de la vida del estado, incluye un amplio catálogo de principios y derechos que se consideran fundamentales, que emanan por lo general de los tratados sobre Derechos Humanos que ha ratificado el Estado, así como de la dignidad misma que como principio persigue siempre el ser humano. (p.429)

Por lo tanto, se resalta la dignidad del ser humanos, al promover los diferentes tratados que vinculan su protección, mediante una serie de principios, pactos, acuerdos y convenios, a nivel nacional e internacional, cuyo objetivo es suscitar las distintas estrategias que permitan el respeto de los derechos humanos de los ciudadanos. Al respecto, según Araujo Landaeta (2016), la Defensoría del Pueblo es un órgano de 


\section{CIENCIAMATRIA}

Revista Interdisciplinaria de Humanidades, Educación, Ciencia y Tecnología

Año VIII. Vol. VIII. №14. Enero - Junio. 2022

Hecho el depósito de ley: pp201602FA4721

ISSN-L: 2542-3029; ISSN: 2610-802X

Universidad Nacional Experimental Francisco de Miranda (UNEFM). Santa Ana de Coro. Venezuela

Rously Eedyah Atencio-González; Elba Tatiana Peñaherrera-Martínez; Rudy Gabriela Yong-Zambrano Gabriel Alexander Lopez-Sanchez

derecho público con jurisdicción nacional, personalidad jurídica y autonomía administrativa y financiera (art. 214), su estructura es desconcentrada y pertenece a la Función de Transparencia y Control Social. Además de tener un papel de veedora para garantizar la transversalización del enfoque de derechos y para que la normativa local se armonice con los instrumentos internacionales, dicha institución puede incidir de manera directa en las etapas de construcción normativa, así como en el ciclo de implementación, monitoreo y evaluación de resultados.

Según Canepa (2015) en el ámbito del MERCOSUR en esta materia:

Ya en el preámbulo los Estados consideran que la protección y garantía de los derechos fundamentales es una condición necesaria para la consolidación del proceso de integración. Los Estados reafirmanlos principios y disposiciones contenidas en distintos instrumentos internacionales de los cuales forman parte, a saber: Declaración Americana de Derechos y Deberes del Hombre, la Convención Americana sobre Derechos Humanos y otros instrumentos regionales de derechos humanos, como la Carta Democrática Interamericana. Al reafirmar las normas contenidas en estos instrumentos internacionales, los Estados están reconociendo que si bien no son parte del derecho mercosureño, los mismos nutren con su espíritu la protección de los derechos humanos en el MERCOSUR. (p.163)

Al interpretar el criterio anterior, se evidencia la búsqueda de extender a todos los ciudadanos, de los diferentes países de Sur los derechos Humanos, es decir cubrir más allá de las fronteras tanto a los internos como los de otras latitudes. A nivel exterior se extienden los beneficios para los ciudadanos ecuatorianos, lo que representa un apoyo que garantice el derecho de los ciudadanos que han emigrado del país, en el 2001 se elaboró el Plan Nacional de Migrantes Ecuatorianos en el Exterior para garantizar los derechos fundamentales de los emigrantes, así como para organizar su residencia en el exterior en condiciones de regularidad, colaborar con la sociedad civil y organismos internacionales para la atención preferencial de familias de ecuatorianos que emigraron, teniendo como objetivo principal reforzar los vínculos con los países receptores, a fin de elaborar acciones coordinadas y conjuntas para diseñar y aplicar programas a favor de 


\begin{abstract}
CIENCIAMATRIA
Revista Interdisciplinaria de Humanidades, Educación, Ciencia y Tecnología

Año VIII. Vol. VIII. N¹4. Enero - Junio. 2022

Hecho el depósito de ley: pp201602FA4721

ISSN-L: 2542-3029; ISSN: 2610-802X

Universidad Nacional Experimental Francisco de Miranda (UNEFM). Santa Ana de Coro. Venezuela

Rously Eedyah Atencio-González; Elba Tatiana Peñaherrera-Martínez; Rudy Gabriela Yong-Zambrano Gabriel Alexander Lopez-Sanchez
\end{abstract}

los emigrados (Serrano, 2006, p.24).

Al respecto Arteaga Cruz (2017) describe lo siguiente:

El Buen Vivir es uno de los conceptos desplegados en estos diez años que apuntaló ideológicamente el discurso de cambio que representaba el gobierno progresista en Ecuador. En el marco de la construcción de nuevas constituciones en la zona andina, el Sumak Kawsay/ Buen Vivir se plasmó en la Constitución del Ecuador de 2008 y continúa siendo parte significativa de los debates a nivel nacional, regional y mundial. (p.908)

Es precisamente el buen vivir, lo que dinamiza y promueve el desarrollo de los valores humanos de los ciudadanos ecuatorianos, amparados en sus normas y leyes establecidas en su carta magna. De acuerdo a los planteamientos formulados se plantea como objetivo general de la investigación analizar la constitucionalidad de los derechos humanos en Ecuador.

\title{
METODOLOGÍA
}

El investigador organizó un proceso investigativo en donde la población de estudio, se basó primordialmente en documentos escritos como normas, leyes. Desde el paradigma cuantitativo, se manejó para este tipo de estudio, documentos, textos legales, investigaciones científicas mediante la recolección, selección y análisis de los mismos. El diseño bibliográfico-documental se apoyó en los procesos lógicos y mentales. Se consideró el análisis de contenido de la información recopilada, lo que permitió la formulación de resultados. Así lo analítico-sintético, consistió en descomponer mentalmente el sistema estudiado en varios elementos para poder llegar a la obtención de nuevos conocimientos del tema investigado. 


\section{CIENCIAMATRIA}

Revista Interdisciplinaria de Humanidades, Educación, Ciencia y Tecnología

Año VIII. Vol. VIII. №14. Enero - Junio. 2022

Hecho el depósito de ley: pp201602FA4721

ISSN-L: 2542-3029; ISSN: 2610-802X

Universidad Nacional Experimental Francisco de Miranda (UNEFM). Santa Ana de Coro. Venezuela

Rously Eedyah Atencio-González; Elba Tatiana Peñaherrera-Martínez; Rudy Gabriela Yong-Zambrano Gabriel Alexander Lopez-Sanchez

\section{RESULTADOS}

Luego de la revisión documental-bibliográfica se presenta a continuación los resultados obtenidos.

\section{Cuadro 1.}

Constitucionalidad de los derechos humanos.

\begin{tabular}{|c|c|}
\hline Institucionalidad-norma & Contenido \\
\hline $\begin{array}{l}\text { Organización de las Naciones Unidas } \\
\text { (1948). }\end{array}$ & $\begin{array}{l}\text { Declaración Universal de los Derechos } \\
\text { Humanos (1948). } \\
\text { Artículo 2.-Toda persona tiene todos los } \\
\text { derechos y libertades proclamados en } \\
\text { esta Declaración, sin distinción alguna de } \\
\text { raza, color, sexo, idioma, religión, opinión } \\
\text { política o de cualquier otra índole, origen } \\
\text { nacional o social, posición económica, } \\
\text { nacimiento o cualquier otra condición. } \\
\text { Artículo 7.-Todos son iguales ante la ley y } \\
\text { tienen, sin distinción, derecho a igual } \\
\text { protección de la ley. Todos tienen derecho } \\
\text { a igual protección contra toda } \\
\text { discriminación que infrinja esta } \\
\text { Declaración y contra toda provocación a } \\
\text { tal discriminación. } \\
\text { Artículo 13.- Parágrafo 1. Toda persona } \\
\text { tiene derecho a circular libremente y a } \\
\text { elegir su residencia en el territorio de un } \\
\text { Estado. } \\
\text { Parágrafo 2. Toda persona tiene derecho } \\
\text { a salir de cualquier país, incluso del } \\
\text { propio, y a regresar a su país. }\end{array}$ \\
\hline $\begin{array}{l}\text { Asamblea Nacional Constituyente de la } \\
\text { República del Ecuador (2008). }\end{array}$ & $\begin{array}{l}\text { Artículo 9.- Las personas extranjeras que } \\
\text { se encuentren en el territorio ecuatoriano } \\
\text { tendrán los mismos derechos y deberes } \\
\text { que las ecuatorianas, de acuerdo con la } \\
\text { Constitución. } \\
\text { Artículo } 40 \text {.-Se reconoce a las personas el } \\
\text { derecho a migrar. No se identificará ni se } \\
\text { considerará a ningún ser humano como }\end{array}$ \\
\hline
\end{tabular}




\begin{tabular}{|l|l|}
\hline & $\begin{array}{l}\text { ilegal por su condición migratoria. } \\
\text { Artículo 41.-Se reconocen los derechos de } \\
\text { asilo y refugio, de acuerdo con la ley y los } \\
\text { instrumentos internacionales de derechos } \\
\text { humanos. Las personas que se } \\
\text { encuentren en condición de asilo o refugio } \\
\text { gozarán de protección especial que } \\
\text { garantice el pleno ejercicio de sus } \\
\text { derechos. El Estado respetará y } \\
\text { garantizará el principio de no devolución, } \\
\text { además de la asistencia humanitaria y } \\
\text { jurídica de emergencia. No se aplicará a } \\
\text { las personas solicitantes de asilo o refugio } \\
\text { sanciones penales por el hecho de su } \\
\text { ingreso o de su permanencia en situación } \\
\text { de irregularidad. El Estado, de manera } \\
\text { excepcional y cuando las circunstancias lo } \\
\text { ameriten, reconocerá a un colectivo el } \\
\text { estatuto de refugiado, de acuerdo con la } \\
\text { ley. } \\
\text { Artículo 392.- El Estado velará por los } \\
\text { derechos de las personas en movilidad } \\
\text { humana y ejercerá la rectoría de la política } \\
\text { migratoria a través del órgano competente } \\
\text { en coordinación con los distintos niveles } \\
\text { de gobierno. El Estado diseñará, } \\
\text { adoptará, ejecutará y evaluará políticas, } \\
\text { planes, programas y proyectos, y } \\
\text { coordinará la acción de sus organismos } \\
\text { con la de otros Estados y organizaciones } \\
\text { de la sociedad civil que trabajen en } \\
\text { movilidad humana a nivel nacional e } \\
\text { internacional. }\end{array}$ \\
\hline Ley \\
Jurisdiccionales y Control Constitucional \\
(2009)
\end{tabular}




\begin{tabular}{|c|c|}
\hline & $\begin{array}{l}\text { pública, hábeas data, por incumplimiento, } \\
\text { extraordinaria de protección y } \\
\text { extraordinaria de protección contra } \\
\text { decisiones de la justicia indígena. } \\
\text { Artículo } 40 .- \text { Requisitos. - La acción de } \\
\text { protección se podrá presentar cuando } \\
\text { concurran los siguientes requisitos: } \\
\text { 1. Violación de un derecho constitucional; } \\
\text { 2. Acción u omisión de autoridad pública o } \\
\text { de un particular de conformidad con el } \\
\text { artículo siguiente; y, } \\
\text { 3. Inexistencia de otro mecanismo de } \\
\text { defensa judicial adecuado y eficaz para } \\
\text { proteger el derecho violado. }\end{array}$ \\
\hline $\begin{array}{l}\text { Organización Internacional de las } \\
\text { Migraciones (2015). }\end{array}$ & $\begin{array}{l}\text { Plantea tres objetivos específicos: } \\
\text { 1. La buena gobernanza de la migración y } \\
\text { las políticas conexas deberían fomentar el } \\
\text { bienestar socioeconómico de los } \\
\text { migrantes y de la sociedad. } \\
\text { 2. La gobernanza adecuada de la } \\
\text { migración se debería fundamentar en } \\
\text { respuestas eficaces a la movilidad en } \\
\text { situaciones de crisis. } \\
\text { 3. La migración se debería efectuar de } \\
\text { manera segura, ordenada y digna. }\end{array}$ \\
\hline
\end{tabular}

Elaboración: Los autores (2022).

Al observar el cuadro 1, se puede apreciar el interés que los Estados y las organizaciones buscan ofrecer garantías a sus ciudadanos sobre los derechos humanos tanto de las personas que se encuentran en el país como los migrantes, ya que la finalidad última es el buen vivir de los pueblos.

Es importante resaltar lo descrito por Araujo Landaeta (2016):

Entre las garantías constitucionales definidas en la Carta Magna (título III), se pueden resaltar las garantías normativas, pues el Estado tiene la obligación de adecuar su ordenamiento jurídico a los derechos humanos contenidos en la Constitución y en los instrumentos internacionales. De igual forma, este 


\section{CIENCIAMATRIA}

Revista Interdisciplinaria de Humanidades, Educación, Ciencia y Tecnología

Año VIII. Vol. VIII. №14. Enero - Junio. 2022

Hecho el depósito de ley: pp201602FA4721

ISSN-L: 2542-3029; ISSN: 2610-802X

Universidad Nacional Experimental Francisco de Miranda (UNEFM). Santa Ana de Coro. Venezuela

Rously Eedyah Atencio-González; Elba Tatiana Peñaherrera-Martínez; Rudy Gabriela Yong-Zambrano Gabriel Alexander Lopez-Sanchez

cuerpo legal prioriza la protección de derechos, afirmando que "la Constitución y los tratados internacionales de derechos humanos ratificados por el Estado que reconozcan derechos más favorables a los contenidos en la Constitución, prevalecerán sobre cualquier otra norma jurídica o acto del poder público". ( $\mathrm{p}$. 13-14)

\section{CONCLUSIÓN}

Al concluir la investigación, se destaca la importancia jurídica y social del derecho constitucional relacionado a la protección de los derechos humanos en el Ecuador y más allá de sus fronteras, gracias a los convenios firmados con los organismos internacionales, como garantista el Estado ha propiciado normas que promueven el buen vivir y por ende el resguardo de los ciudadanos.

Gracias al análisis jurídico realizado, se evidenció que los derechos humanos desde el punto de vista legal y social, por su reconocimiento constitucional cobra vital importancia en vigencia y aplicación por parte del Estado ecuatoriano y sus órganos competentes.

\section{FINANCIAMIENTO}

No Monetario.

\section{AGRADECIMIENTO}

A la Universidad Regional Autónoma de los Andes, Quevedo; por motivar el desarrollo de la investigación.

\section{REFERENCIAS CONSULTADAS}

Araujo Landeta, P., Pacheco Posso, D., y Redrobán Herrera, V. (2016). Contribuciones de la Defensoría del Pueblo de Ecuador para la transversalización del enfoque de derechos humanos en la normativa local. [Contributions of the Ombudsman's Office of Ecuador for the mainstreaming of the human rights approach in local regulations] Defensoría del Pueblo Ecuador. Recuperado de: https://n9.cl/q09la 


\section{CIENCIAMATRIA}

Revista Interdisciplinaria de Humanidades, Educación, Ciencia y Tecnología

Año VIII. Vol. VIII. №14. Enero - Junio. 2022

Hecho el depósito de ley: pp201602FA4721

ISSN-L: 2542-3029; ISSN: 2610-802X

Universidad Nacional Experimental Francisco de Miranda (UNEFM). Santa Ana de Coro. Venezuela

Rously Eedyah Atencio-González; Elba Tatiana Peñaherrera-Martínez; Rudy Gabriela Yong-Zambrano Gabriel Alexander Lopez-Sanchez

Arteaga Cruz, E. (2017). Buen Vivir (Sumak Kawsay): definiciones, crítica e implicaciones en la planificación del desarrollo en Ecuador. [Good Living (SumakKawsay): definitions, critique and implications for development planning in Ecuador]. SAÚDE DEBATE RIO DE JANEIRO, 41(114), 907-919. DOI: 10.1590/01031104201711419

Asamblea Nacional (2009).Ley Orgánica de Garantías Jurisdiccionales y Control Constitucional. [Organic Law on Jurisdictional Guarantees and Constitutional Control]. Ley 0 Registro Oficial Suplemento 52 de 22-oct-2009. Recuperado de: https://n9.cl/su7v

Asamblea Nacional Constituyente de la República del Ecuador, (2008). Constitución de la República del Ecuador. [Montecristi. Registro Oficial 449 de 20-oct-2008. Recuperado de https://n9.cl/sia

Canepa, M. (2015). Los derechos humanos en el MERCOSUR.[ Human rights in MERCOSUR]. Revista de la Secretaría del Tribunal Permanente de Revisión, 3(6), 161-177. https://dx.doi.org/10.16890/rstpr.a3.n6.161

Lema, M. (2009) Derechos humanos y acceso a la justicia en Ecuador.[ Human rights and access to justice in Ecuador] Instituto Interamericano de Derechos Humanos San José de Costa Rica. Recuperado de: https://n9.cl/nfhcm

OIM. (2015). Marco de la Gobernanza de la Migración. [Migration Governance Framework]. Ginebra. Recuperado de: https://bit.ly/2RVy008

Organización de las Naciones Unidas (1948). La Declaración Universal de Derechos Humanos [The Universal Declaration of Human Rights]. Recuperado de https://n9.cl/xe66

Serrano, J. (2006) Trabajadores migrantes: Protección de sus derechos laborales y programas de mercado de trabajo. [Migrant workers: Protection of their labour rights and labour market programmes] Ponencia presentada en Ottawa, Canadá, 28 y 29 de noviembre. 


\section{CIENCIAMATRIA}

Revista Interdisciplinaria de Humanidades, Educación, Ciencia y Tecnología

Año VIII. Vol. VIII. N¹4. Enero - Junio. 2022

Hecho el depósito de ley: pp201602FA4721

ISSN-L: 2542-3029; ISSN: 2610-802X

Universidad Nacional Experimental Francisco de Miranda (UNEFM). Santa Ana de Coro. Venezuela

Rously Eedyah Atencio-González; Elba Tatiana Peñaherrera-Martínez; Rudy Gabriela Yong-Zambrano Gabriel Alexander Lopez-Sanchez

Sosa Salazar, E., Campoverde Nivicela, L., y Sánchez Cuenca, M. (2019). Los principios de titularidad, exigibilidad e igualdad y no discriminación como principios de aplicación de los derechos en el Estado ecuatoriano. [The principles of ownership, enforceability and equality and non-discrimination as principles of application of rights in the Ecuadorian State]. Revista Universidad y Sociedad, 11(5), 428-436. Recuperado de: https://n9.cl/gar01

C2022 por los autores. Este artículo es de acceso abierto y distribuido según los términos y condiciones de la licencia Creative Commons Atribución-NoComercial-Compartirlgual 4.0 Internacional (CC BY-NC-SA 4.0) (https://creativecommons.org/licenses/by-nc-sa/4.0/). 